\title{
Eye Gaze Patterns Differentiate Novice and Experts in a Virtual Laparoscopic Surgery Training Environment
}

\author{
Benjamin Law \\ School of Computing Science \\ Simon Fraser University
}

\author{
M. Stella Atkins* \\ School of Computing Science \\ Simon Fraser University
}

\author{
A.E. Kirkpatrick \\ School of Computing Science \\ Simon Fraser University
}

\author{
Alan J. Lomax \\ School of Kinesiology \\ Simon Fraser University
}

Christine L. Mackenzie

School of Kinesiology

Simon Fraser University

\begin{abstract}
Visual information is important in surgeons' manipulative performance especially in laparoscopic surgery where tactual feedback is less than in open surgery. The study of surgeons' eye movements is an innovative way of assessing skill, in that a comparison of the eye movement strategies between expert surgeons and novices may show important differences that could be used in training. We conducted a preliminary study comparing the eye movements of 5 experts and 5 novices performing a one-handed aiming task on a computer-based laparoscopic surgery simulator. The performance results showed that experts were quicker and generally committed fewer errors than novices. We investigated eye movements as a possible factor for experts performing better than novices. The results from eye gaze analysis showed that novices needed more visual feedback of the tool position to complete the task than did experts. In addition, the experts tended to maintain eye gaze on the target while manipulating the tool, whereas novices were more varied in their behaviours. For example, we found that on some trials, novices tracked the movement of the tool until it reached the target.
\end{abstract}

Keywords: eye tracking, laparoscopic surgery, virtual training environment

\section{Introduction}

Minimally invasive surgery (MIS) is less traumatic to a patient than in open surgery because only three to four small incisions are required instead of one large wound, and recovery times are greatly reduced. An example of how MIS has improved patient outcomes is in open cholecystectomies (gall bladder removals) which can take up to four to six days for the patient to recover, but laparoscopic surgery has reduced this recovery time to an overnight stay at the hospital [Gelijns and Rosenberg 1995]. There are several types of MIS such as arthroscopy, endoscopy and laparoscopy. We focus on MIS in the abdominal area or also known as laparoscopy. Cholecystectomy is an example of a laparoscopic procedure. In MIS, the surgeon inserts narrow diameter laparoscopic tools into small incisions

*e-mail:stella@cs.sfu.ca on the patient whose internal abdominal cavity is inflated with carbon dioxide to increase working space. To see inside the abdominal area, another tool called a laparoscope is inserted into the patient through another small incision. A laparoscope is a tube with one end containing a lens surrounded by a fibre optic bundle that transmits light from a halogen or xenon light source to avoid burning tissue with the light source. This fibre optic bundle illuminates the abdominal cavity, and a camera mounted on the laparoscope captures images so that internal body structures and the end-effectors of laparoscopic tools are visible on a video monitor. See Figure 1 for an illustration of how the laparoscopic tools and laparoscope are situated on a patient's abdominal area. The laparoscope is usually controlled by another member of the surgical team, such as a resident [Eyal and Tendick 2001; Tendick et al. 1998].

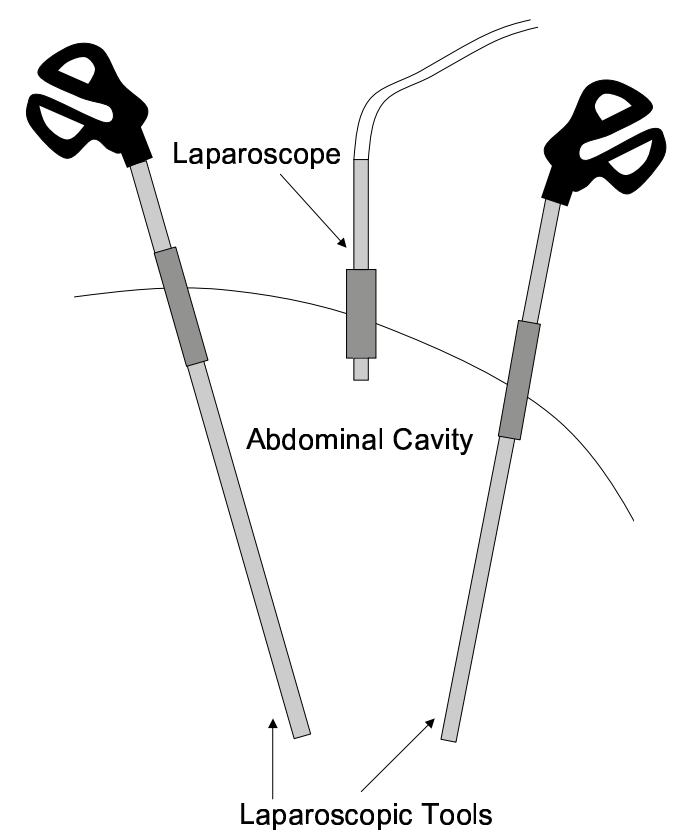

Figure 1: Illustration of how laparoscopic instruments enter a patient's body. 


\subsection{Minimally Invasive Surgery: Visuomotor Issues}

Laparoscopic procedures require more care and skill on the part of the surgeon than open surgery [Gallagher and Satava 2002]. Thus, there is interest in developing effective training systems for laparoscopy. For example, tool movement appears to move in the opposite direction of hand movement; this phenomenon is known as the "fulcrum effect" [Gallagher et al. 2001] and has been shown to negatively affect novices performing laparoscopic tasks [Jordan et al. 2001]. The fulcrum effect also reduces dexterity because the fulcrum point restricts tool movement, and thus, arbitrary tool movement is not possible [Tendick et al. 2000].

Another difference between open and minimally-invasive surgeries is that visual and work spaces are not located in the same area, which leads to errors and increased manipulation time [Cuschieri 1995; Ibbotson et al. 1999; Tendick and Cavusoglu 1997]. Laparoscopic tool movement is unlike direct hand movement because proprioceptive feedback from hand position does not map directly to the tool tips [Smith et al. 2000] necessitating additional visuomotor and spatial transformations [MacKenzie et al. 1999]. Tactile feedback from tool movement is minimal because of friction between the tool and the cannula (a tunnel like structure surrounding the tool at the patient entry point), and thus, the surgeon has a greater reliance on the indirect visual information [Cuschieri 1995; Ibbotson et al. 1999].

New laparoscopic surgeons must overcome these challenges and traditional training devices include training boxes or live animal surgeries. Computer based simulation trainers offer a flexible alternative. The resident can practice at anytime. The validation of these virtual laparoscopic training systems are currently underway and already a virtual laparoscopic training system has been shown to differentiate skill [Gallagher and Satava 2002] using measures such as time, number of errors, and economy of tool motion. This training system is not a complete recreation of an actual surgical procedure, but instead relies on smaller tasks or part-tasks to train specific skills [Payandeh et al. 2002]. Such systems are simpler to develop and their effectiveness in training surgeons [Seymour et al. 2002] influenced our decision to design a simple hand-eye co-ordination task for an eye-tracker experiment.

\subsection{Eye Movement Differences in Experts and Non- Experts}

In other domains such as radiology, differences in search strategies between novices and experts have been found [Nodine and MelloThoms 2000]. Nodine and Mello-Thoms cited a study that showed the time to hit a target (i.e. a lesion) was shorter for experienced mammographers than observers with less experience and training. In another cited study, experienced radiologists tended to use a circumferential scan pattern and avoided scanning the edges of the lung tissues, but less experienced observers were attracted to the edges of the lungs. A possible cause for this behaviour is the expert knowledge that lesions are less likely to be found in the edges of the lungs.

Another study [Kasarskis et al. 2001] showed differences in performance and eye movements between expert and novice pilots who performed landings in a flight simulator. Predictably, expert pilots landed better than novices, but they also found that dwell times in experts were shorter than novices indicating that experts gathered the required information quicker than novices. The two groups also differed in the distribution of fixation locations. Experts fixated more frequently on the airspeed indicator, but fewer fixations on the altimeter, during landing. On the other hand, novices fixated more frequently on the altimeter. The experts' fixation behaviour is learned by their knowledge that the airspeed indicator was more informative.
These results show that domain knowledge and experience affect performance and eye movements on a related task. Knowledge and hand-eye co-ordination ability are both important in MIS, and we continue to look at additional studies on eye movement differences between subjects with different levels of skill in hand-eye co-ordination tasks.

One example of eye movement differences is from Vickers [Vickers 1995] who showed eye gaze pattern differences between expert and near-expert collegiate-level basketball players in a foul shooting task. Experts tended to end their fixations earlier in the shot suggesting that the visual system was used to program the motor system. Novices, on the other hand, used visual input to adjust their shots until the ball was off the hand. In another sport, Vickers [Vickers 1993] also compared eye movements of low and high handicap golf putters. Expert golfers tended to perform fewer eye movements between different locations to minimize memory decay of distance cues [Vickers 1993].

The eye gaze differences between low and high skilled individuals suggest that another way to measure skill could be to use eye gaze measures and behaviours in an eye-hand co-ordination task. Laparoscopic surgery is an interesting area of study to use eyetrackers because of the constraints on the visuomotor system as described in Section 1.1. We hypothesise that there are eye gaze differences between novices with no prior experience of laparoscopic procedures and experts who have experience with laparoscopic surgery. This paper is an initial attempt to compare the eye movement behaviour of these groups during performance of a laparoscopic surgery-like task.

\section{Method}

\subsection{Participants}

Ten right-handed subjects, that consisted of five novices and five experts, participated in the study. The novice subjects were students at Simon Fraser University. All expert subjects had experience with laparoscopic surgery; four were surgeons at a local hospital, and one retired from active practice. All participants had normal or corrected to normal vision. One subject wore glasses. There was one female in each group.

\subsection{Materials}

Subjects were seated (see Figure 2(a)) on a non-adjustable chair while viewing a 17" Dell TFT monitor set at 1280x1024 resolution with a dot pitch of $0.264 \mathrm{~mm}$. The monitor was connected to a PC with an AMD Athlon 1.2GHz processor and an Asus V6800 32MB video card. Subjects used an Immersion Corp. Laparoscopic Impulse Engine to control the a virtual tool displayed on the screen. Tool position data were saved to a log file, sampled at $50 \mathrm{~Hz}$.

An ASL 504 remote eye tracker was used to sample the subjects' eye gaze at $60 \mathrm{~Hz}$. The data were sent to a PC through a serial connection and saved to an ASCII log file. Each eye gaze sample was averaged over 4 fields using the ASL control software to smooth out small-magnitude eye gaze position jitter. The eye tracker control software ran on an IBM 390E laptop. A Focus Enhancements TView Gold 300 scan converter was used to create a composite video frame of eye gaze location overlayed on the scene (i.e., the frame captures of the experimental task). The experimental task was implemented as a Visual C++ application using OpenGL.

\subsection{Experimental Task}

Subjects performed a virtual aiming task (see Figure2(b)), developed from a pilot study [Law et al. 2003], with the goal of reaching for and touching a small target with a virtual tool tip controlled by 


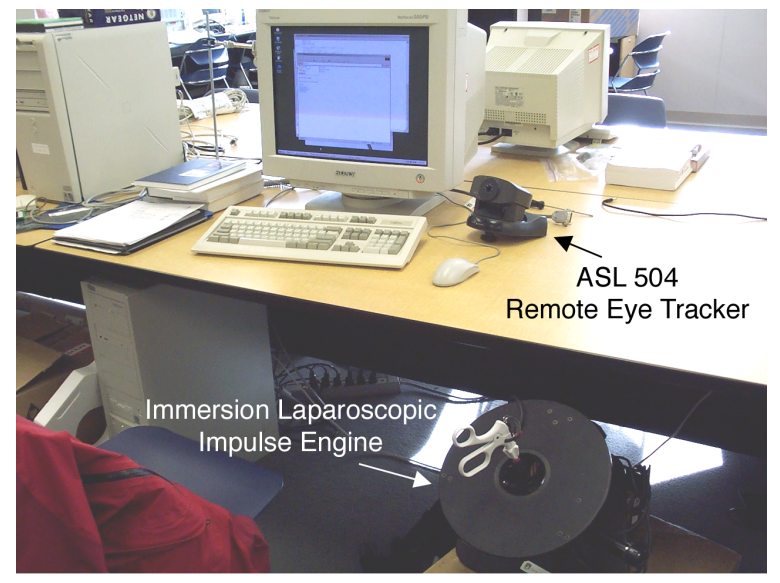

(a) Experimental set up

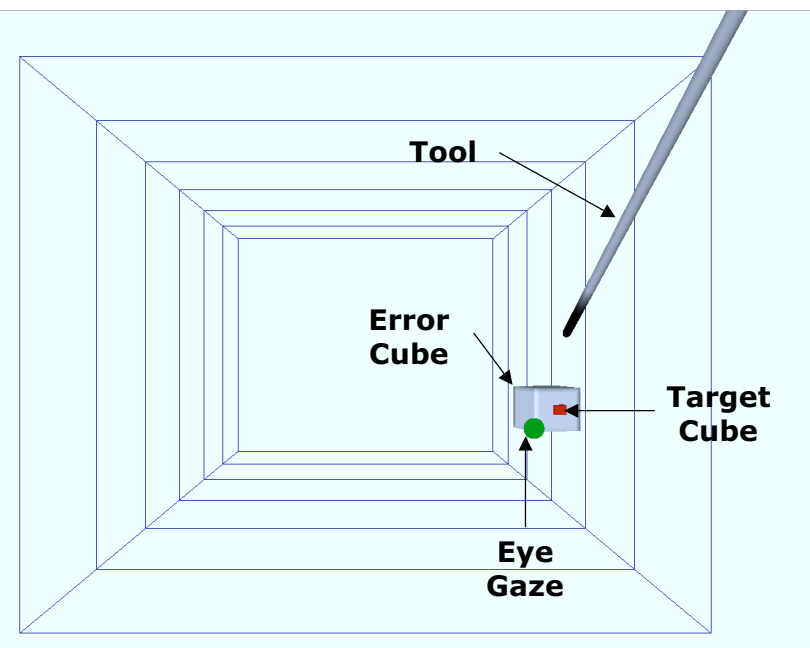

(b) Screen shot of aiming task

Figure 2: Experimental materials

the Laparoscopic Impulse Engine. Similar one-handed tasks have been developed for assessing surgical skills [Hanna et al. 1996] and studying the role of visual cues in virtual aiming performance [Tendick et al. 2000]. In our task, the tool tip had to touch the surface of the virtual target cube before the trial was considered complete. If the tool end tip touched the surface of the larger cube surrounding the target cube, an error was recorded, and the surface changed colour. Tool movement was restricted at a pivot point (the point was positioned beyond the viewing volume) but the tool could rotate around the point and translate in and out of the pivot point. When the tool end tip made contact with the target cube, a new trial started by clearing the old target from the screen and displaying a new target at another location on the screen. Haptic feedback was not provided, in order to avoid confounding the response measures. The experimental task was presented so that interposition of the tool and the target provided a monocular depth cue. Other depth cues that subjects could extract from the virtual environment were the relative size of the tool itself and the linear perspective provided by the background. Subjects were asked to complete the task as quickly and as error free as possible.

\subsection{Procedure}

Subjects remained seated at a distance of approximately $80 \mathrm{~cm}$ from the monitor. Subjects started with two practice trials. After completion of the practice trials, their eyes were calibrated for the eye tracker. Eye calibration requires subjects to fixate on each point of a 9 point grid displayed on the computer monitor. After calibration, subjects were asked to re-fixate on each point on the grid and the eye gaze locations were saved to a log file. This data was used to compute the calibration accuracy.

Each subject performed 2 blocks of 5 trials, with a short break allowed between blocks. After completing a block, subjects were asked to fixate again on each of the 9 points on the calibration grid. Eye gaze locations were saved to a log file to calculate the accuracy of the eye gaze recordings during the experiment. Both groups filled out a questionnaire after finishing all the blocks.

\subsection{Data Collection}

Two ASCII log files are produced over the course of an experiment. The first file contains the data samples from the eye tracker. Recorded in parallel with the eye tracker log file is the file containing samples of the tool location. Both log files contain a timestamp indicating when the log file started recording data and another timestamp for each sample record. Note that these log files are updated and stored on the same computer so that clock differences between computers can be ignored. Before data analysis can begin, our application, LTEEYE, synchronizes the tool position and eye gaze co-ordinates using the timestamps. LTEEYE synchronizes the data in two ways depending on what type of analysis is necessary. For qualitative analysis of the data where the experiment is replayed on screen, the application reads each tool position sample with its associated timestamp and then determines the corresponding eye gaze after factoring in latency from data transport and data averaging. Because the eye gaze data are in a different co-ordinate system than the screen co-ordinates, each eye gaze sample is transformed into screen co-ordinates [Duchowski and Vertegaal 2000] so that LTEEYE can render the eye gaze position along with the scene of the user's tool movements in the virtual environment (see Figure2(b)). The result is a video replay of the experimental session where each frame is re-rendered using the tool and eye gaze positions data. The replay can be suspended, reversed, or slowed down to facilitate analysis.

The synchronization method described above, however, fail to take into account some eye gaze samples because the gaze $(60 \mathrm{~Hz})$ and tool position $(50 \mathrm{~Hz})$ sampling rates are different. To ensure that all eye gaze and tool events are included in the analysis, LTEEYE combines and serializes all records in both eye gaze and user tool $\log$ files. Each record is as an event that updates the scene state; either the eye gaze or tool positions in the scene are modified depending on the event. This synchronization method requires more computational time than the previous method because more frames are rendered, but allows more accurate quantitative analysis as will be discussed in Section 2.6. To answer the question, "Was the subject looking at the tool or the target?", we mapped the points-ofregard (raw eye gaze data) to the elements-of-regard (the graphical objects rendered on screen [Reeder et al. 2001]). This mapping, also known as points-to-elements mapping [Reeder et al. 2001], is performed in our application by defining a picking region centered around the eye gaze point to determine what scene elements were rendered in this region. This picking region was a square centred on the eye gaze point to approximate the foveal field of view. The width of this square subtends a visual angle of $2.5^{\circ}$. The mapping procedure was run after the experiments were completed to minimize computational load while the experimental task was running. 


\subsection{Data Analysis}

The performance measures were the total time to complete each block of aiming task trials, and the total number of errors committed in each block. Eye gaze measures included accuracy, and the gaze time on the tool. The accuracy was used to eliminate data where subjects significantly moved their heads [Law 2003]. The gaze time on the tool was expressed as a proportion of total time. Longer gaze times on the tool indicated greater visual feedback was needed on the tool position and movement, particularly when the tool and the target were not within foveal vision. The gaze times on tool were calculated by combining and serializing both eye and user tool samples as described in Section 2.5 and then performing the pointsto-elements mapping at each event. By summing the durations of each event where the element-of-regard was the tool, we obtained the total gaze duration on the tool. We also examined the movement profiles to categorize the trajectories of the tool and eye gaze.

\section{Results and Discussion}

\subsection{Performance Measures}

Figures 3(a) and 3(b) show the mean completion times and mean number of errors respectively for both groups, along with the standard deviations.

The frequency plots, shown in Figures 4(a) and 4(b), indicate that the data for both time and errors have non-normal distributions. Thus, we used the two sample Kolmogorov-Smirnov non-parametric test to analyze the raw data. The data were combined across blocks before applying the test. The analysis showed that experts were significantly quicker than novices $(Z=1.581, p=0.013)$, but experts did not commit significantly fewer errors than novices $(Z=0.949, p=0.329)$. We also analyzed the time and error data (transformed with natural logarithm function) using a $2 \times 2$ (Group $\times$ Block) mixed ANOVA with repeated measures on the blocks factor. The experts overall completed the task significantly quicker than the novices $(F(1,8)=23.594, p=0.001)$. The ANOVA test for errors yielded a difference approaching significance $(F(1,8)=4.92, p=0.057)$ between experts and novices, with the experts committing fewer errors than novices. Thus, both tests showed that experts performed significantly quicker than novices, but did not show significance for the errors measure.

The performance differences show that the experts performed better than novices with no minimally-invasive surgery experience, and that this simple virtual aiming task can be used to differentiate task skill between the two groups. Thus, the following eye gaze analysis may be associated with task performance, and in the next section we investigate eye movements as a possible factor of task skill.

\subsection{Eye Gaze Measures}

Eye gaze behaviour for this task can be categorized into two main ways: average distribution of gaze location and co-ordination patterns of gaze and tool movement over the visual display.

\subsubsection{Gaze Distributions}

We measured the amount of time the eye gaze was on the tool area of interest. A foveal region is defined by an area centred at the eye gaze point. If the foveal region and the tool position overlapped, and the target was not within the foveal region at that time, then a gaze on tool was recorded. Table 1 shows the amount of time subjects gazed only on the tool as a proportion of their completion time per block.

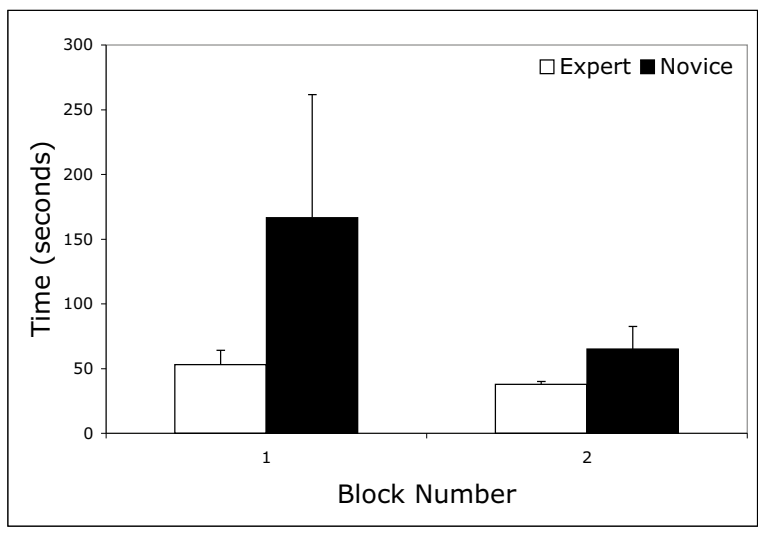

(a) Mean completion times

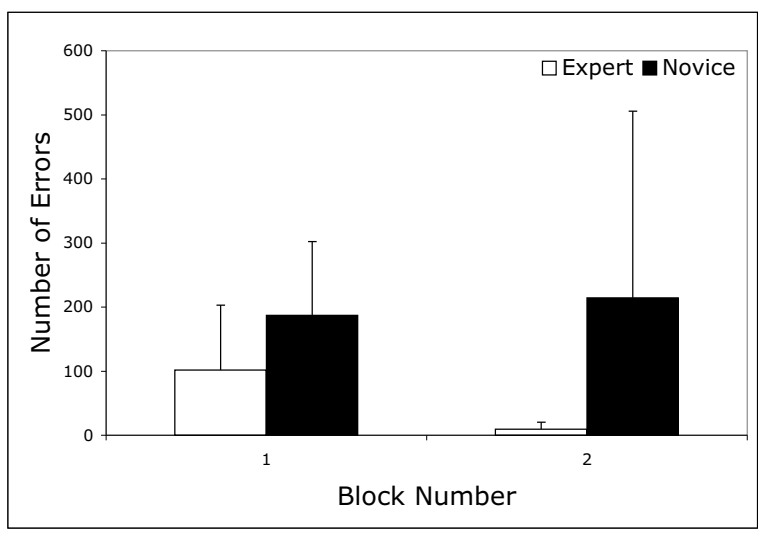

(b) Mean number of errors

Figure 3: Performance measures for expert and novice groups, shown with 1 standard deviation

Although the arithmetic means showed group differences in the proportion of eye gaze duration on the tool, we used trimmed means to remove outliers that could affect the values given their small values. From Table 1, even with $30 \%$ of outer values trimmed (i.e. the lower and upper $15 \%$ of scores were discarded and then the remaining values were used to compute the mean), the difference of means between groups is $2.16 \%$ whereas the difference between the groups' arithmetic means yield a difference of $2.83 \%$. Thus, group differences still remain even when outliers are removed. This difference was expected because novices were unfamiliar with coordinating their hand with tool movements. Running the results through an ANOVA, however, yielded non-significant differences. Figure 5 shows a box plot of the eye gaze duration on the tool as a proportion of block completion time for blocks 1 and 2. The figure

Table 1: Proportion of eye gaze on tool for blocks 1 and 2.

\begin{tabular}{c|c|c}
\hline Measure & Expert & Novice \\
\hline Mean & $2.29 \%$ & $5.12 \%$ \\
$10 \%$ Trim Mean & $2.29 \%$ & $5.12 \%$ \\
$30 \%$ Trim Mean & $2.29 \%$ & $4.45 \%$
\end{tabular}




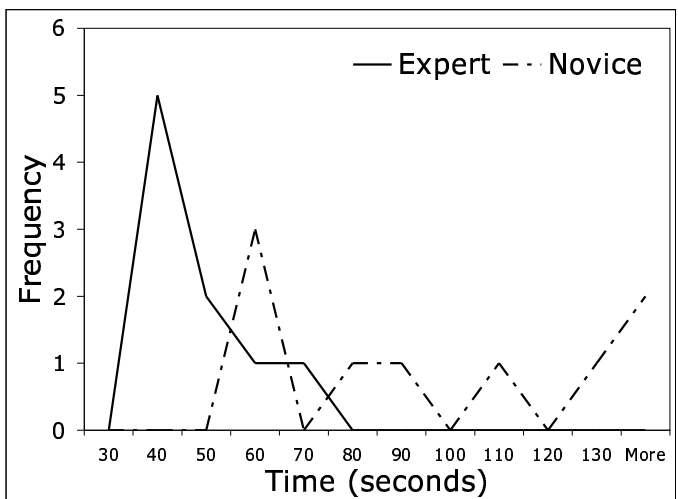

(a) Time dependent measure

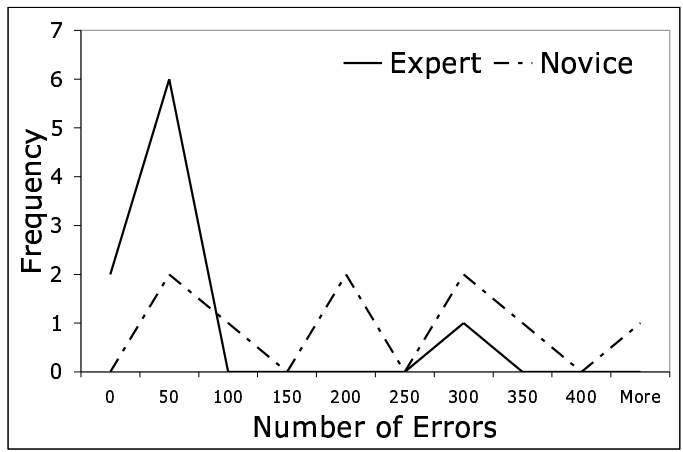

(b) Error dependent measure

Figure 4: Frequency plots of time and error measures for each group

shows that despite the group differences, the data points in the expert group are contained within $75 \%$ of values in the novice group.

Average gaze location suggests some differences in gaze behaviour regarding the tool. We now examine the gaze movement patterns over time with respect to the tool.

\subsubsection{Gaze Movement Profiles}

Gaze movement profile charts were created to display eye gaze and tool distances to the target centre with respect to time. Figures 69 are examples of such profiles. We found several eye-hand coordination behaviours in our subjects similar to those found in a previous study [Smith et al. 2000]. The dark line in each chart represents the distance of the eye gaze to the target centre, and the lighter line represents the distance of the tool tip to the target centre. Note that the tool tip can be quite close to the target in this graph, but far along the viewing axis. The y-axis on these figures only measures eye gaze distance from the target. Thus, one cannot determine the direction of eye movement from simply the distance between the eye gaze and target. To determine if the saccades were indeed toward the tool and not to a random screen location, the distance between tool tip and eye gaze was plotted against time. These plots (not shown) indicate that the saccades of interest were always toward either the target or tool in Figures 6-9.

One gaze movement behaviour that we found from observing the experimental replays was target gaze behaviour, illustrated in

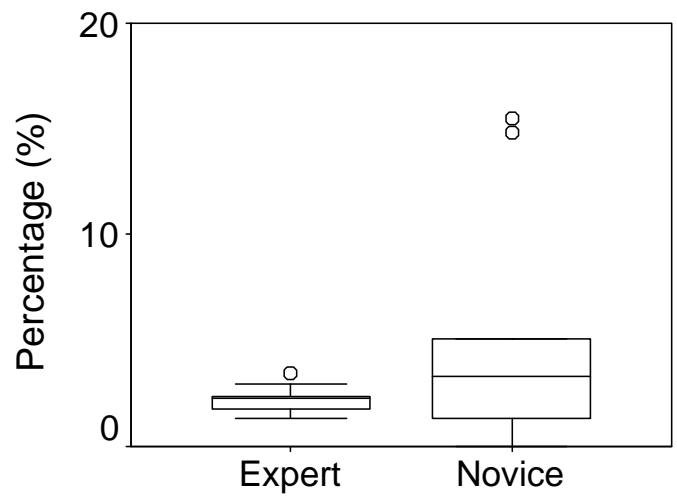

Group

Figure 5: Boxplot of proportion of eye gaze on tool for blocks 1 and 2.

Figure 6 . Before the tool reached the vicinity of the target, eye gaze was already on target location and did not deviate away from the target after the initial saccade to the target.

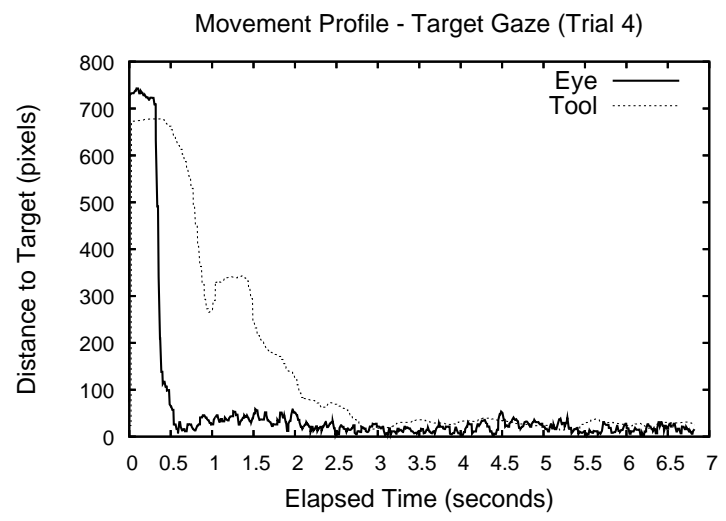

Figure 6: Target gaze behaviour from an expert subject (S1)

A switching behaviour, shown in Figure 7, started with the eye gaze moving to the target. The subject performed a saccade to the tool (verified by plotting distance between tool and eye gaze location over time) after about 1.5 seconds which is shown on the movement profile chart as a decrease in the slope of the dashed curve. The foveal view was on the tool for a short time (about 0.3 seconds), and then another saccade was made back to the target. Presumably the subject at that point needed information about target location that could not be acquired using peripheral vision. As in the target gaze behaviour described above, the location of eye gaze was on the target well before the tool was in the vicinity of the target.

Another similar type of switching behaviour was observed in the data where the saccade end point was located in an area between the target and the tool tip, rather than at the tip itself. This type of "tool leading" behaviour is illustrated in Figure 8. Only a portion of the trial (4.5 to 10 seconds) is plotted on the figure due to the lengthy trial duration. The saccade started at approximately 6.3 seconds when the tool tip was distant from the target centre (about 580 pixels) and ended at a location about 200 pixels, or $53 \mathrm{~mm}$ away from the target centre. The eye gaze remained at this location while the 


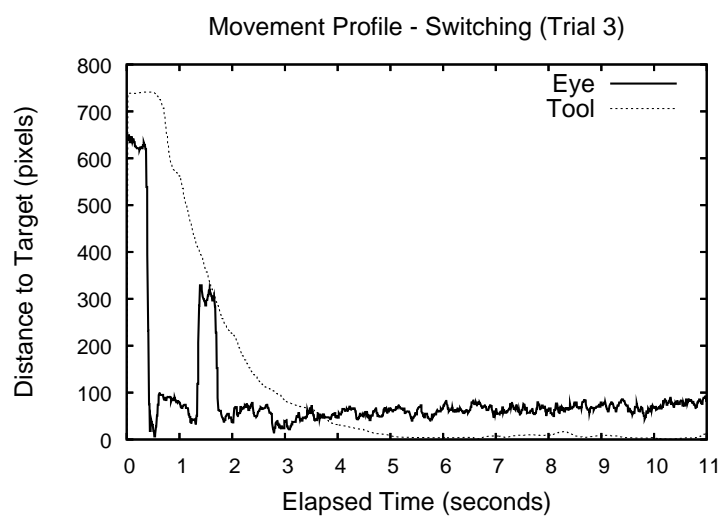

Figure 7: Switching behaviour - on tool from novice subject (S7)

tool tip moved into foveal view, suggesting that the eye gaze was leading the tool towards the target.

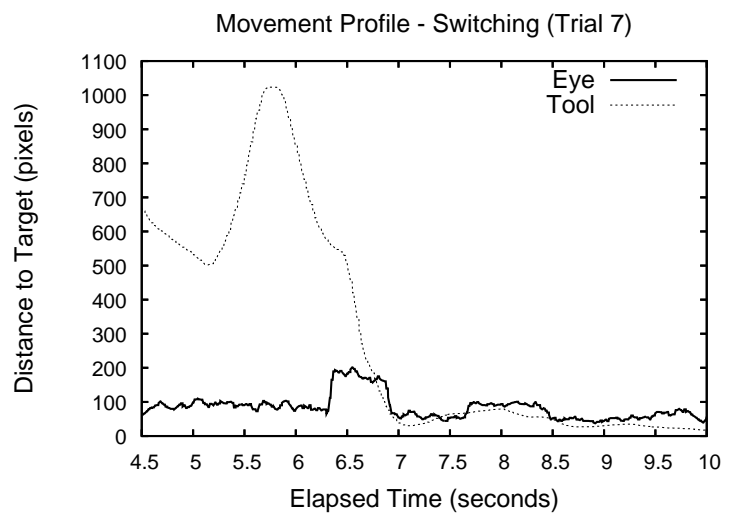

Figure 8: Switching behaviour - part way towards tool from novice subject (S7)

Another eye-hand co-ordination strategy was a tool following behaviour. The eye gaze appeared to be tracking the tool tip while it was moving. An example is shown in Figure 9 where the subject is gazing at the tool along the path from the start to the end points. The eye gaze does not reach within 100 pixels, or about $26 \mathrm{~mm}$, of the target until approximately 3 seconds after the start of the trial.

The replays of all trials were observed to categorize them into one of the previously described behaviours. Behaviours were coded as follows: A trial was categorized as a target gaze behaviour when the point of eye gaze arrived at the target before the tool tip. In addition, the eye gaze stayed on target for the remainder of the trial, and the eye gaze made no saccades to the tool. In trials with switching behaviour, at least one occurrence of a saccade from the target to the tool must occur. Trials with tool following behaviour are characterized by tool movements that are tracked by the eye. This eye and tool movement pattern can be seen when the tool and eye gaze were near the target, and then the tool moved away from the target followed by the eye gaze. Tool following was also coded when the eye gaze was on the target, but the tool was positioned outside the foveal view; a subject would make a saccade to the tool and then eye gaze tracked the tool as it moved towards the target. Tool following could also be observed at the start of the trial when the eye gaze and tool reach the target simultaneously as illustrated in Fig-

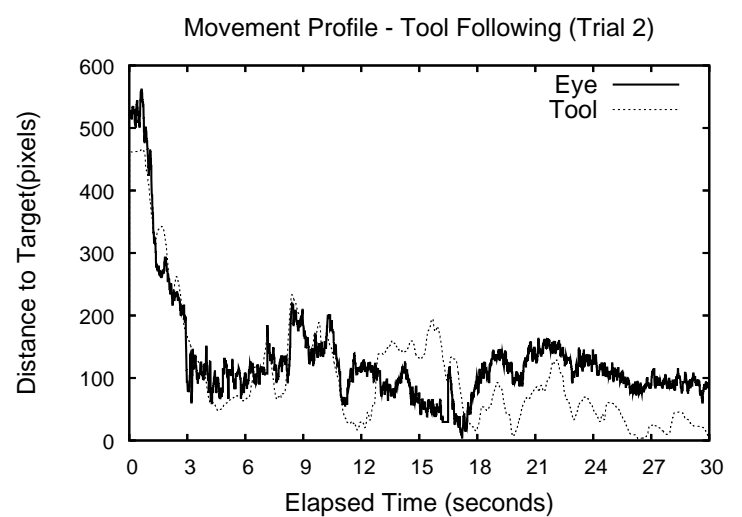

Figure 9: Tool following behaviour from a novice subject (S9)

Table 2: Eye movement behaviour distributions for expert and novices over all trials. Expert subjects engaged in target gaze behaviour more frequently and tool following less frequently than novices.

\begin{tabular}{c|c|c|c|c}
\hline Group & Target Gaze & Switching & Tool Follow & Loss \\
\hline Expert & $73.3 \%$ & $13.3 \%$ & $8.9 \%$ & $4.4 \%$ \\
Novice & $53.3 \%$ & $17.8 \%$ & $26.7 \%$ & $2.2 \%$
\end{tabular}

ure 9. Trials were categorized as loss when no eye gaze data was available in the replay from the start of the trial until the tool was near the target. The missing eye gaze data was caused by eye blinks or the eye tracker lost either pupil or corneal reflection signals.

The results after categorizing each trial are shown in Table 2. Expert surgeons and novices had distinctly different eye gaze behaviour distributions. There was a slight difference between the two groups in the frequency of switching behaviour with the novices $(17.8 \%)$ employing the switching behaviour slightly more often than experts $(13.3 \%)$. The other behaviours, target gaze and tool following, show larger differences between groups. Experts engaged in target gaze behaviour $(73 \%)$ more frequently than novices $(53.3 \%)$, but engaged in tool following $(8.9 \%)$ less frequently than novices $(26.7 \%)$. Overall, the experts had a stronger preference to engage in target gaze behaviour. The strategies for novices varied as more novices engaged in tool following. The novices needed more visual feedback than the experts because of their unfamiliarity with tool movement, and this behaviour could also be part of the learning process to map the hand and tool movements.

The movement profile figures (Figures 6-9) show long "tails" where both tool and eye gaze were close to the target at the last phase of the trial. Recall that these movement profiles only show the 2-D Euclidean distance between the tool and the target and not their distance along the viewing axis. With this in mind, these tails indicate a strategy where subjects made an initial tool movement similar to moving a mouse cursor over a 2-D monitor. After the initial movement, the position of the tool tip was in front of the target. The tool was then moved towards the target along the viewing axis while fine movements of the tool were made to maintain it over the target position on the $\mathrm{x}-\mathrm{y}$ plane. This two-phase approach to the aiming task was confirmed in subject responses from the questionnaire asking subjects to describe their strategy or approach to the aiming task. All subjects in both groups employed this strategy indicating that eye gaze behaviour differences occurred primarily in the initial phase of tool movement. The eye movement strategies of novices who needed foveal vision to guide the tool to the target sug- 
gest they had not yet mastered tool manipulation in the initial phase. This would also suggest that novices would have more difficulty in the phase when they "home in" on the target with the tool.

\section{Summary and Future Work}

This preliminary user study on eye movements in a laparoscopic training system compared experts and novices in a virtual aiming task. The results showed performance and eye movement differences between the two groups. The experts were quicker, and our results show a trend that they were more accurate than novices. To see if the performance differences between groups were accompanied with eye movement differences, we looked at the amount of eye gaze on the tool and then characterized their eye behaviour through eye and tool movement profiles. In terms of eye gaze behaviour, novices tended to gaze at the tool longer than experts. Several eye gaze behaviours identified in this study, including target gaze, switching, and tool following, are similar to previous findings [Smith et al. 2000]. The target gaze behaviour was the preferred strategy for experts, and novices tended to tool follow more frequently than experts. These eye movement differences suggest that it may be possible to assess the skills of surgeons as part of a battery of tests, and it could be used to assess the progress of training surgeons.

In the future, we plan to collect eye gaze data in more difficult two-handed laparoscopic surgery-like tasks such as cutting and suturing in a virtual environment or a physical training box. Such tasks would yield richer data on the eye movement differences between novices and expert surgeons and possibly differences between skill-levels within the expert group (although this may be harder to show given the difficulty in obtaining a large number of experienced surgeons).

\section{Acknowledgments}

Funding for this work was provided by the Canada Natural Science and Engineering Research Council. The authors would also like to thank Dr. Shahram Payandeh for the code which the application was based and for loaning some of the hardware used in the user experiments.

\section{References}

CUSCHIERI, A. 1995. Visual displays and visual perception in minimal access surgery. Seminars in Laparoscopic Surgery 2, 3, 209-214.

Duchowski, A., AND VertegaAl, R. 2000. SIGGRAPH 2000 Course 05 Notes. Eye-Based Interaction in Graphical Systems: Theory and Practice.

EYAL, R., AND TENDICK, F. 2001. Spatial ability and learning the use of an angled laparoscope in a virtual environment. In Proceedings of Medicine Meets Virtual Reality (MMVR), IOS Press, 146-153.

Gallagher, A., AND SataVa, R. 2002. Virtual reality as a metric for the assessment of laparoscopic psychomotor skills. Surgical Endoscopy $16,12,1746-1752$.

Gallagher, A., Richie, K., McClure, N., And McGuigan, J. 2001. Objective psychomotor skills assessment of experienced, junior, and novice laparoscopists with virtual reality. World Journal of Surgery $25,1478-1483$

Gelijns, A., And Rosenberg, N. 1995. From the scalpel to the scope: Endoscopic innovations in gastroenterology, gynecology, and surgery. In Sources of Medical Technology: Universities and Industry, N. Rosenberg, A. Gelijns, and H. Dawkins, Eds., vol. V of Medical Innovation at the Crossroads. National Academy Press.
Hanna, G., Drew, T., Clinch, P., Hunter, B., Schimi, S., DunKLEY, M., AND CUSCHIERI, A. 1996. A micro-processor controlled psychomotor tester for minimal access surgery. Surgical Endoscopy 10, 965-969.

Ibbotson, J., MacKenzie, C., CaO, C., And LomaX, A. 1999. Gaze patterns in laparoscopic surgery. In Proceedings of Medicine Meets Virtual Reality (MMVR), IOS Press, 154-160.

Jordan, J., Gallagher, A., McGuigan, J., And McClure, N. 2001. Virtual reality training leads to faster adaptation to the novel psychomotor restrictions encountered by laparoscopic surgeons. Surgical Endoscopy 15, 1080-1084.

Kasarskis, P., Stehwien, J., Hickox, J., ARETZ, A., AND WickENS, C. 2001. Comparison of expert and novice scan behaviors during VFR flight. In Proceedings of the 11th International Symposium on Aviation Psychology. http://www . aviation.uiuc.edu/UnitsHFD/ conference/proced01.pdf.

Law, B., Atkins, M. S., Lomax, A., And Wilson, J. 2003. Eye trackers in a virtual laparoscopic training environment. In Proceedings of Medicine Meets Virtual Reality (MMVR), IOS Press, 184-186.

LAw, B. 2003. Eye Movements in a Virtual Laparoscopic Training Environment. Master's thesis, Simon Fraser University, School of Computing Science. ftp://fas.sfu.ca/pub/cs/theses/2003/ BenjaminLawMSc.pdf.

MacKenzie, C., Graham, E., CaO, C., and LomaX, A. 1999. Virtual hand laboratory meets endoscopic surgery. In Proceedings of Medicine Meets Virtual Reality (MMVR), IOS Press, 212-218.

Nodine, C., AND Mello-Thoms, C. 2000. The nature of expertise in radiology. In Handbook of Medical Imaging, J. Beutel, H. Kundel, and R. Van Metter, Eds. SPIE Press.

Payandeh, S., Lomax, A., Dill, J., MacKenzie, C., and Cao, C. 2002. On defining metrics for assessing laparoscopic surgical skills in a virtual training environment. In Proceedings of Medicine Meets Virtual Reality (MMVR), IOS Press, 334-340.

Reeder, R., Pirolli, P., ANd CARD, S. 2001. WebEyeMapper and WebLogger: Tools for analyzing eye tracking data collected in web-use studies. In CHI 'O1 Extended Abstracts on Human factors in Computer Systems, 19-20.

Seymour, N., Gallagher, A., Roman, S., O'Brien, M., Bansal, V., ANDERSEN, D., AND SATAVA, R. 2002. Virtual reality training improves operating room performance: Results of a randomized, doubleblinded study. Ann Surg 236, 4, 458-463.

Smith, B., Ho, J., ARK, W., AND ZHAI, S. 2000. Hand eye coordination patterns in target selection. In Proceedings of ACM Symposium of Eye Tracking Research and Applications (ETRA), 117-122.

Tendick, F., ANd CAvusoglu, M. 1997. Human-machine interfaces for minimally invasive surgery. In Proceedings of the 19th Annual International Conference of the IEEE Engineering in Medicine and Biology Society, 2771-2776.

Tendick, F., Downes, M., Cavusoglu, M., Gantert, W., And WAY, L. 1998. Development of virtual environments for training skills and reducing errors in laparoscopic surgery. In Proceedings of the SPIE International Symposium on Biological Optics (BIOS '98), 36-44.

Tendick, F., Downes, M., Goktekin, T., Cavusoglu, M., Feygin, D., Wu, X., Eyal, R., Hegarty, M., AND Way, L. 2000. A virtual environment testbed for training laparoscopic surgical skills. Presence 9, 3, 236-255.

VICKERS, J. 1993. Toward defining the role of gaze control in complex targeting skills. In Visual Search, D. Brogan, G. A., and K. Carr, Eds. Taylor and Francis, Ltd.

VICKERS, J. 1995. Gaze control in basketball foul shooting. In Eye Movement Research: Mechanisms, Processes, and Applications, J. Findlay, R. Walker, and R. Kentridge, Eds. Elsevier, 527-541. 